\title{
The effect of pilocarpine on the glaucomatous visual field
}

\author{
Andrew R Webster, Andrew J Luff, Christopher R Canning, Andrew R Elkington
}

\begin{abstract}
Patients with chronic open angle glaucoma are traditionally managed by medical therapy during the early stages of the disease. Pilocarpine is a well established topical agent, but suffers troublesome sequelae, the most apparent of which is pupillary constriction. This study assesses the effect of miosis (produced by one drop of $2 \%$ pilocarpine) on the static threshold perimetry of 20 subjects with chronic open angle glaucoma and documented visual field loss, using the 30-2 program of the Humphrey field analyser. Following miosis, the Statpac mean defect deteriorated by an average of $-1.49 \mathrm{~dB}$ compared with baseline $(p=0.004)$. This $\mathrm{dB}$ deterioration is twice that reported in studies on younger normal subjects following miosis. The decrease in mean defect showed a positive correlation with the degree of pupillary constriction, the correlation being greater in those eyes with a miosed pupil diameter of $2 \mathrm{~mm}$ or less. There was no significant decrease in the other Statpac global indices following miosis. A parallel study using the fellow eye of the same glaucoma patients showed a high degree of intertest variability, but no significant learning or fatigue effect. We conclude that pilocarpine-induced miosis causes a significant deterioration in visual field in a population of patients with chronic open angle glaucoma: this factor should be considered when choosing therapy for glaucoma particularly in cases where field loss approaches the permitted legal minimum for driving.

(Brf Ophthalmol 1993; 77: 721-725)
\end{abstract}

Pilocarpine is an important topical agent in the treatment of glaucoma, a condition in which perimetry plays a pivotal role in diagnosis and management. ${ }^{1-3}$ Pilocarpine diminishes visual fields in normal subjects as measured using both kinetic and static perimeters. ${ }^{45}$ It is clinically relevant to determine whether glaucomatous patients have a different, or exaggerated, response to pilocarpine when compared with previous results on normal subjects.

This study assesses the effect of pilocarpine on full threshold visual field testing in glaucomatous patients with pre-existing field loss. It also examines the intertest variability of such patients during field testing, and determines any learning or fatigue effects that might occur on consecutive testing.

Patients and methods

Twenty volunteers were recruited from patients with chronic open angle glaucoma (COAG) attending Southampton Eye Hospital. All patients were on medical therapy, or had previously undergone trabeculectomy, and had glaucomatous field loss on Goldmann or Humphrey perimetry. Patients already on miotic therapy were excluded, as were those with normal perimetry, other potential causes of field defects or advanced media opacity. Other groups excluded were those with a Snellen visual acuity of less than 6/36 in either eye, those incurring many fixation losses (greater than 20\%), and those with many false positives or negatives (greater than 33\%) on the Humphrey field analyser (HFA).

Written informed consent was obtained from each patient entering the study, local ethical committee approval having been granted. One eye of each patient was designated the 'study eye' and the other the 'fellow eye' by the investigators. Often if the field loss was particularly asymmetrical, the eye with the greater field loss was chosen as the study eye. At no time in the analysis of the data were the results from each eye compared directly, and the allocation of the study eye was not and did not need to be undertaken randomly.

A baseline examination included refraction for near ( $1 / 3$ metre $)$ and distance, corrected near and distance acuities, slit-lamp examination, and biomicroscopic funduscopy. The baseline pupil diameter was measured using the HFA monitor (magnification factor 1.5 ) to the nearest $0.5 \mathrm{~mm}$ at the test background luminance of 31.5 apostilbs (10 Nit). Each subject, if not familiar with the HFA, underwent a short practice session. Four 30-2 full threshold tests were performed, two on each eye, on the same day. The study eye underwent a baseline test, and then a second test 30 to 40 minutes after instillation of one drop (from a minim) of $2 \%$ pilocarpine. The fellow eye underwent two tests without pilocarpine. Before each test, refractive error for near ( $1 / 3$ metre) was corrected using the Humphrey trial frame. Before testing the miosed eye, the pupil diameter measurement and refraction for near were repeated and the optical correction modified as required. The order of the test sequence was alternated between subjects such that the study eye was analysed first in half of the group. The subject was observed closely during the tests and any pseudo-loss of fixation was recognised and remedied as has been previously described. ${ }^{6}$ The four Statpac global indices, mean defect (MD), pattern standard deviation (PSD), short term fluctuation (SF), and corrected pattern standard deviation (CPSD), were used in subsequent analysis. The results from the study eye were used in the determination of the effect of miosis, and those from the fellow eye in the determination of
Accepted for publication 15 June 1993 
Table 1 Pupil diameter measurements

\begin{tabular}{|c|c|c|c|c|c|}
\hline & \multicolumn{3}{|c|}{ Study eye pupil (mm) } & \multicolumn{2}{|c|}{ Fellow eye pupil $(\mathrm{mm})$} \\
\hline & Baseline & Miosed & Difference & Ist test & 2nd test \\
\hline $\begin{array}{l}\text { Mean } \\
\text { Range }\end{array}$ & $\begin{array}{l}5 \cdot 5 \\
2 \cdot 7-7 \cdot 7\end{array}$ & $\begin{array}{l}2 \cdot 0 \\
1 \cdot 0-3 \cdot 3\end{array}$ & $\begin{array}{l}3 \cdot 5 \\
1 \cdot 3-5 \cdot 3\end{array}$ & $\begin{array}{l}5 \cdot 5 \\
2 \cdot 7-8 \cdot 7\end{array}$ & $\begin{array}{l}5 \cdot 5 \\
3 \cdot 0-7 \cdot 3\end{array}$ \\
\hline
\end{tabular}

learning or fatigue effects and variability. A nonalgebraic mean value of the difference in MD of the two tests on the fellow eye was calculated thus and was taken as an indication of the long term or intertest variability in this group of glaucomatous subjects:

$$
\Sigma_{\mathrm{I}=1}^{20} \frac{|M D 2 \mathrm{i}-\mathrm{MD} 1 \mathrm{i}|}{20}
$$

MDni=mean defect during nth test of ith subject.

\section{Results}

Of the 20 subjects, 14 were male. The mean age was 70.6 (SD 9.3) years with a range of 48-83 years.

Pupil diameter measurements are shown in Table 1. Although the mean pupil diameter of the fellow eyes did not change between the first and second tests, individual fluctuations occurred with seven pupils dilating (range 0.3 to 1.0 $\mathrm{mm}$ ), five constricting (range 0.3 to $2.0 \mathrm{~mm}$ ), and eight remaining the same. Two patients had anisocoria of greater than $2 \mathrm{~mm}$ : one was using dipivalyl adrenaline unilaterally and the other had a marked relative afferent pupil defect, the pupil diameter being measured with the non-test eye occluded. Eighteen patients were found to have an unchanged refraction for near following miosis. One became $1 \cdot 25$ dioptres myopic (48 years) and one became 1.0 dioptre hypermetropic. Of the 40 eyes at baseline examination, the visual acuities were: 30 eyes $6 / 9$ or better, four eyes $6 / 12$, two eyes $6 / 18$, three eyes $6 / 24$, and one eye $6 / 36$. Of the four eyes seeing $6 / 24$ or worse, only one incurred greater than $20 \%$

Table 2 Baseline global indices

\begin{tabular}{|c|c|c|c|c|c|c|}
\hline \multirow{2}{*}{$\begin{array}{l}\text { Index } \\
(d B)\end{array}$} & \multicolumn{2}{|c|}{ All eyes $(n=40)$} & \multicolumn{2}{|c|}{ Study eye $(n=20)$} & \multicolumn{2}{|c|}{ Fellow eye $(n=20$} \\
\hline & Mean & $S D$ & Mean & $S D$ & Mean & $S D$ \\
\hline $\begin{array}{l}\text { MD } \\
\text { PSD } \\
\text { SF } \\
\text { CPSD }\end{array}$ & $\begin{array}{r}-12.42 \\
8.07 \\
2.41 \\
7.41\end{array}$ & $\begin{array}{l}8 \cdot 88 \\
3 \cdot 75 \\
1 \cdot 19 \\
3 \cdot 90\end{array}$ & $\begin{array}{r}-15 \cdot 58 \\
9.91 \\
2 \cdot 87 \\
9 \cdot 20\end{array}$ & $\begin{array}{l}7 \cdot 47 \\
2 \cdot 77 \\
1 \cdot 16 \\
2 \cdot 99\end{array}$ & $\begin{array}{r}-9.26 \\
6.23 \\
1.94 \\
5.61\end{array}$ & $\begin{array}{l}9.22 \\
3.76 \\
1.06 \\
3.93\end{array}$ \\
\hline
\end{tabular}

$M D=$ mean defect.

$P S D=$ pattern standard deviation

$\mathrm{SF}=$ short term fluctuation.

$\mathrm{CPSD}=$ corrected pattern standard deviation

Table 3 Changes in global indices in study eye (moised minus baseline) and fellow eye (second test minus first test) for all 20 subjects, and those with good miosis (pupil constricting to $2 \mathrm{~mm}$ or less): $p$ values calculated using paired Student's t test (two tailed)

\begin{tabular}{|c|c|c|c|c|c|c|c|c|}
\hline & \multicolumn{4}{|c|}{ All subjects $(n=20)$} & \multicolumn{4}{|c|}{ Subjects with good miosis $(n=13)$} \\
\hline & \multicolumn{2}{|c|}{ Study eye } & \multicolumn{2}{|c|}{ Fellow eye } & \multicolumn{2}{|c|}{ Study eye } & \multicolumn{2}{|c|}{ Fellow eye } \\
\hline & Mean & $p$ & Mean & $p$ & Mean & $p$ & Mean & $p$ \\
\hline $\begin{array}{l}\text { MD } \\
\text { PSD } \\
\text { SF } \\
\text { CPSD }\end{array}$ & $\begin{array}{r}-1.49 \\
0.45 \\
0.24 \\
0.5\end{array}$ & $\begin{array}{l}0.004 \\
0.134 \\
0.498 \\
0.351\end{array}$ & $\begin{array}{l}-0.72 \\
-0.47 \\
-0.32 \\
-0.31\end{array}$ & $\begin{array}{l}0 \cdot 102 \\
0.094 \\
0 \cdot 371 \\
0.406\end{array}$ & $\begin{array}{c}-1.73 \\
0.6 \\
-0.08 \\
0.93\end{array}$ & $\begin{array}{l}0.006 \\
0.083 \\
0.866 \\
0.204\end{array}$ & $\begin{array}{l}-0.56 \\
-0.67 \\
-0.22 \\
-0.55\end{array}$ & $\begin{array}{l}0.333 \\
0.048 \\
0.614 \\
0 \cdot 298\end{array}$ \\
\hline
\end{tabular}

For all global indices, a minus sign indicates a deterioration.
Table 4 Deterioration in MD according to degree of miosis p Values calculated using paired Student's t test (two tailed)

\begin{tabular}{|c|c|c|c|}
\hline & $\begin{array}{l}\text { All study eyes } \\
(n=20)\end{array}$ & $\begin{array}{l}\text { Pupil diameter } \\
2 \mathrm{~mm} \text { or less } \\
(\mathrm{n}=13)\end{array}$ & $\begin{array}{l}\text { Pupil diameter } \\
\text { over } 2 \mathrm{~mm} \\
(\mathrm{n}=7)\end{array}$ \\
\hline $\begin{array}{l}\text { Mean pupil } \\
\text { diameter }(\mathrm{mm}) \\
\text { Fall in } M D(\mathrm{~dB}) \\
\text { p Value }\end{array}$ & $\begin{array}{l}1.98 \\
1.49 \\
0.004\end{array}$ & $\begin{array}{l}1.61 \\
1.73 \\
0.006\end{array}$ & $\begin{array}{l}2.62 \\
1.04 \\
0.314\end{array}$ \\
\hline
\end{tabular}

fixation losses (16/62) and this was remedied by relocation of the blind spot. ${ }^{6}$ In those subjects with scotomata adjacent to the blind spot the fixation test was not adequate, and these patients were observed closely using the monitor. In none of the tests on the 20 subjects did false positive or negative responses equal or exceed $33 \%$ (the manufacturer's recommended maximum value for a reliable test).

The initial global indices of the 40 eyes, with breakdown into study eyes and fellow eyes, are shown in Table 2 . The mean MD of the study eyes before miosis was $-15.58 \mathrm{~dB}$, and of the fellow eyes $-9 \cdot 26 \mathrm{~dB}$. Of the 20 study eyes receiving pilocarpine, 17 showed a deterioration in $M D$, with a range of -0.39 to $-4.69 \mathrm{~dB}$. An improvement in MD was seen in three eyes, the range being 1.24 to $1.75 \mathrm{~dB}$. These three subjects included the youngest and second youngest in the study (ages 48 and 49), neither of whom constricted to a pupil diameter of $2 \mathrm{~mm}$. The third had severe glaucomatous field loss in the study eye, with a baseline MD of $-24 \cdot 23 \mathrm{~dB}$.

The changes in the global indices in the study eyes following miosis, and in the fellow eyes between the first and second tests, are shown in Table 3. The mean MD following miosis deteriorated by $-1.49 \mathrm{~dB}$ and was statistically significant $(p=0.004)$. There was a nonsignificant deterioration in $M D$ of $-0.72 \mathrm{~dB}$ $(p=0 \cdot 102)$ in the fellow eyes. The PSD following miosis improved by $0.45 \mathrm{~dB}$ overall but was not significant at the $5 \%$ level $(p=0 \cdot 134)$. The nonalgebraic mean value of the difference in $M D$ of the two tests on the fellow eye was found to be $1.45 \mathrm{~dB}$ and was taken as an indication of the average intertest variability in the 20 glaucomatous subjects in this study.

Thirteen of the 20 study eyes achieved a 'good miosis', constricting to pupil diameters of $2 \mathrm{~mm}$ or less. Analysis of this subgroup showed a slightly greater absolute loss of retinal sensitivity with a mean deterioration in $M D$ of $-1.73 \mathrm{~dB}$ $(p<0.006)$, as seen in Table 4. The mean defect of the fellow eyes decreased by $-0.56 \mathrm{~dB}$, but was not statistically significant $(p=0 \cdot 333)$.

The deterioration in MD of the study eye was examined for correlation with the decrease in pupil diameter, using the product moment correlation coefficient. This showed a correlation coefficient of $0.62(p=0.004)$ for all 20 eyes, and $0.76(p=0.004)$ for those eyes with 'good miosis'. Similar analysis using the pupillary area difference gave similar results, with a correlation coefficient of $0.52(p=0.022)$ for all 20 eyes and $0.80(p=0.002)$ in those eyes with 'good miosis', the latter being illustrated in Figure $1(n=12$ as baseline pupil diameter measurement was lost on one subject).

The suspicion of an inverse relationship 
Figure 1 The relationship between degree of miosis and change in mean defect in subjects with good miosis.

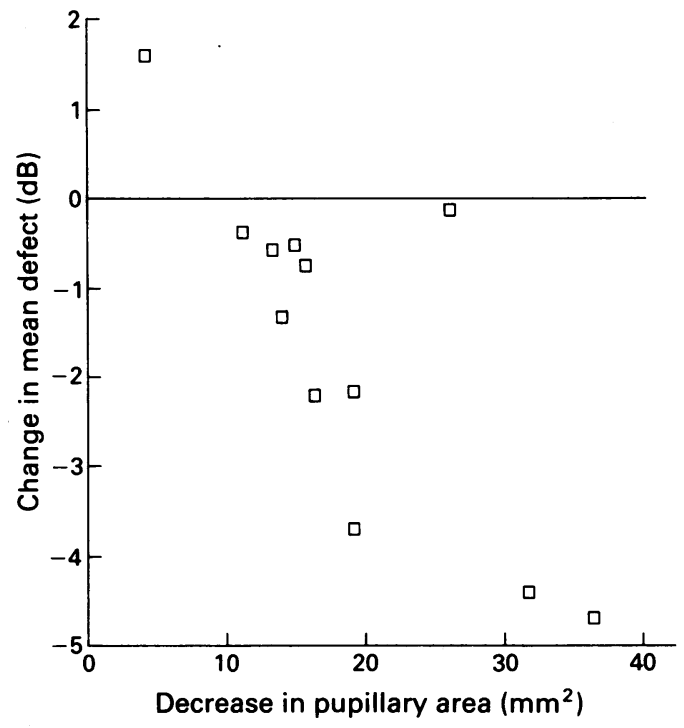

between baseline MD and degree of deterioration following miosis was explored. The mean baseline $M D$ was -19.49 (SD 5.73) $\mathrm{dB}$ in eyes suffering a less than average drop in MD with miosis, and -9.71 (SD 5.84) dB in eyes suffering a greater than average drop in MD with miosis (the average drop being $-1.49 \mathrm{~dB}$ ). The product moment correlation coefficient between deterioration in MD and baseline MD for all 20 study eyes was calculated to be -0.51 ( $\mathrm{p}=$ $0 \cdot 023$ ). When restricted to the 13 eyes with ' $\operatorname{good}$ miosis', a more marked inverse correlation, $-0.83(p<0.001)$, was seen (Fig 2). For statistical purposes an estimation of baseline MD was taken as $1 / 2$ baseline $M D+1 / 2$ miosed $M D$ (see Discussion).

\section{Discussion}

Despite the documented deterioration in the visual field of normal subjects following miosis, little is known of the effect on the glaucomatous patient. Engel showed that physostigmine constricted the visual field to a $5 \mathrm{~mm}$ red (but not white) target at $300 \mathrm{~mm} .^{7}$ Forbes documented cases of glaucomatous patients on miotic therapy

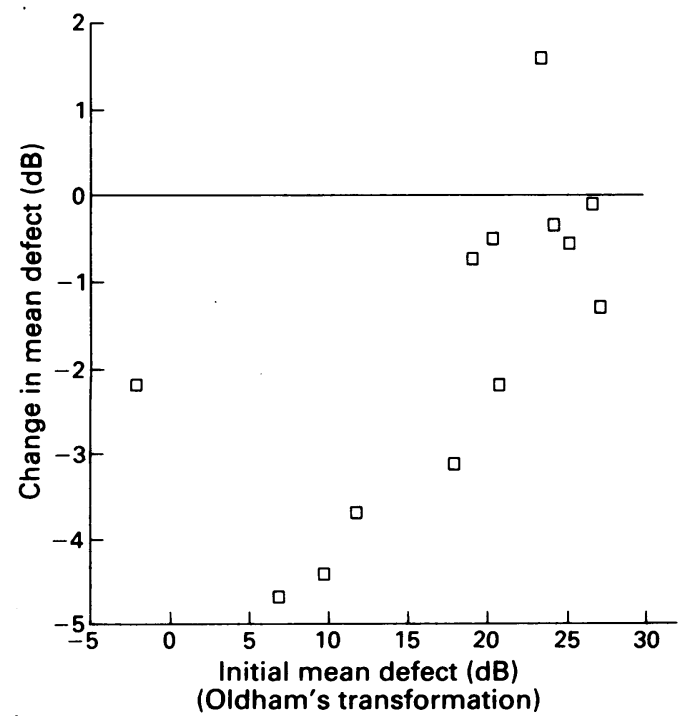

Figure 2 The relationship between baseline mean defect and change in mean defect in subjects with good miosis. whose fields improved with pupillary dilatation, ${ }^{8}$ and more recently Rebolleda et al reached similar conclusions with patients on pilocarpine showing a $3.14 \mathrm{~dB}$ improvement in $\mathrm{MD}$ following dilatation with $10 \%$ phenylephrine. ${ }^{9}$

Certain physiological principles would predict a deterioration in visual function with decreasing pupil size: firstly, retinal illumination falls as the pupil constricts, illumination being approximately proportional to pupillary area; and secondly, increasing miosis results in diffraction of light at the pupil margin, reducing the quality of the retinal image. Conversely, there are three principal physiological considerations that may explain an improvement in visual function with decreasing pupil size. Firstly, a smaller pupil might increase threshold sensitivity by reducing image blurring due to spherical and chromatic aberrations. Secondly, the depth of focus would increase, reducing the blur circles of any ametropia (the pinhole effect). Thirdly, for a given degree of retinal illumination a more effective visual response would result from a reduction in the Stiles-Crawford effect, as light traverses a miosed pupil closer to the visual axis. Indeed, mydriasis (as well as miosis) in normal subjects has been found to reduce the MD on computerised field testing. ${ }^{10}$ The positive and negative effects of pupillary constriction are thought to balance out at an optimal pupil size of $2.4 \mathrm{~mm}$. ${ }^{11}$ The effective area of the pupil is also reduced at greater perimetric angles, but this effect is small and would be negligible when examining the central 30 degrees of the visual field. 1213

The effect of change in retinal illumination should be considered with regard to Weber's law:

$$
\Delta \mathrm{S}=\frac{\Delta \mathrm{I}}{\mathrm{I}}
$$

in which $S$ is a measure of sensation and $I$ is a measure of stimulus intensity. For a threshold sensation the change in stimulus required would vary in proportion to the background stimulation; the brighter the background, the brighter the stimulus required:

$$
\text { Weber-Fechner fraction }=\frac{\Delta \mathrm{I}}{\mathrm{I}}=\text { constant }
$$

This law has been found to apply widely in sensory neurophysiological systems. In the context of perimetry, if the law holds then a reduction in pupillary area would reduce both the stimulus and the background intensity by the same factor and the threshold sensitivity would subsequently remain unchanged. However, evidence suggests that at low levels of retinal illumination, particularly at the mesopic and scotopic range of retinal adaptation, the WeberFechner fraction increases such that a proportionately larger stimulus is required to achieve threshold sensation. This effect has been studied using neutral density filters, a decrease in retinal illumination resulting in deterioration in threshold sensitivity on the Octopus perimeter..$^{14}$ The HFA ( 31.5 apostilbs) has a background illumination nearly one log unit greater than that of the Octopus (4 apostilbs), so such a tendency would be much less marked. 
This study showed a deterioration of -1.49 $\mathrm{dB}$ in mean threshold sensitivity of glaucomatous eyes following miosis. Lindenmuth et al, in a similar study on normal eyes, showed a deterioration in $\mathrm{MD}$ of $-0.67 \mathrm{~dB}$ following miosis. ${ }^{4}$ However, the mean age of the normal subjects in this study was 28.9 years (range 24 to 43 ), and in ours 70.6 years (range 48 to 83 ). The HFA, when calculating the global indices, compares the retinal threshold values at each point in the field with that of a $\mathrm{F}$ pulation of age-matched normals. This would, to some extent, allow comparison of the global indices of the two studies. However, it remains a possibility that older individuals are more susceptible to a change in retinal sensitivity following miosis and this may be a factor in the difference in the findings in the two studies. To separate the two factors of age and glaucoma on the effect of miosis on visual field would require further study. The age range of subjects in this study was thought to be typical of patients affected by COAG who receive miotic treatment and therefore more appropriate when investigating this side effect in the usual clinical setting.

It must be remembered that retinal sensitivity measured on the HFA is expressed on a decilog scale; a given change in MD represents the same proportional base unit change in any patient, irrespective of the baseline MD. Thus the $-1 \cdot 49$ $\mathrm{dB}$ change in MD shown in this study corresponds to a $29 \%$ base unit decrease in retinal threshold sensitivity.

In a study by Quigley et al, histological findings were correlated with previous perimetry in glaucomatous eyes showing that a $20 \%$ to $40 \%$ loss of nerve fibres occurred in areas of retina that corresponded to a 5-10 dB loss of sensitivity. ${ }^{15}$ We were interested to determine whether such areas of retina would be more vulnerable to a decrease in retinal illumination or to an increase in retinal blurring, and that such an eye would show an exaggerated response to miosis. This potential effect is best estimated by reference to the PSD, which responds to changes in the shape of the hill of vision rather than to a uniform change in retinal sensitivity. In this study the PSD did not significantly deteriorate following miosis, which suggests that those areas of retina more highly compromised by glaucoma did not show an exaggerated decrease in sensitivity. Indeed, a mean improvement of 0.45 was observed in the PSD following miosis, although this was not statistically significant. Therefore, the PSD compensates adequately for the effect of miosis. The PSD has been shown to be equally useful in interpreting the reduction in $M D$ due to media opacity ${ }^{16}$ or pupillary dilatation. ${ }^{10}$

The sensitivity of automated perimetry in the detection of true field loss is limited by short term fluctuation, or intratest variability (as measured by the SF global index) and long term fluctuation, or intertest variability. Such intertest variability has been found to be greater in older age groups, ${ }^{17}$ and in patients with glaucoma $^{18}$ or suspected glaucoma. ${ }^{19}$ The interpretation of the clinical significance of any change in sensitivity in this study must be made with caution. A parallel study on the fellow eyes of our subjects showed no significant learning or fatigue effects; a non-significant mean deterioration in the second test compared with the first was found in MD $(-0.72 \mathrm{~dB})$ and PSD $(-0.47$ $\mathrm{dB})$. The value of $-0.72 \mathrm{~dB}$ is greater than the mean deterioration found in normals by Lindenmuth et al (-0.67). It must be remembered that, in the study by Lindenmuth $e t a l$, this was a statistically significant finding in a population of normals whose results had a small dispersion. The glaucomatous patients in our study had a widely dispersed range of results, which therefore needed to show a greater difference in mean values for a given level of significance $(p=$ $0 \cdot 102$ for fellow eye $M D$, see Table 3 ). This wide dispersion in our glaucomatous patients was confirmed by a calculated non-algebraic average value for the variability in $M D$ of $1.45 \mathrm{~dB}$, suggesting that an average subject would be expected to have a $1.45 \mathrm{~dB}$ difference in MD between the first and second tests, which may be either an 'improvement' or a 'deterioration'.

Further analysis of the deterioration in MD following miosis has been made by constructing two subgroups on the basis of the degree of miosis. Analysis of those eyes with pupil diameters of $2 \mathrm{~mm}$ or less ('good miosis') after pilocarpine was performed as any deleterious effect should be more marked with pupil diameters less than $2.4 \mathrm{~mm}$. Table 4 shows that the mean drop in MD in the 13 eyes with 'good miosis' is $-1.73 \mathrm{~dB}$, and remains statistically significant $(p=0.006)$. The decrease in pupil size showed a positive correlation with deterioration in MD $(r=0.62, p=0.006)$, the effect being greater in those eyes with 'good miosis' $(r=0.76$, $\mathrm{p}=0.004$ ), and the coefficient being slightly higher when pupil area was considered $(r=0.52$, $\mathrm{p}=0.022$ and $r=0.8, \mathrm{p}=0.002$ ).

The results suggest that the deterioration in MD following miosis varied directly with the baseline MD. Extrapolation of this observation to examine the correlation between baseline MD and change in MD following miosis is not straightforward. Any attempt to correlate a 'change in value' against 'initial value' is apt to produce a spurious correlation as a result of interdependence of the variables being plotted..$^{20}$ This can be minimised by statistical manipulation, substituting $1 / 2$ initial value+ $1 / 2$ final value' for 'initial value' (Oldham's transformation). When this is done, a weak negative correlation of $-0.51 \quad(p=0.023)$ is demonstrated between change in MD and baseline MD. Applying the same criteria to those eyes with 'good miosis' produced a correlation coefficient of $-0.83(p<0.001)$. Despite the mathematical and statistical uncertainties, it remains a pertinent clinical observation that in this study, the deterioration in MD was greater in those eyes with less marked field loss.

The deterioration in visual field of the miosed eye documented in this study should be considered by all those routinely prescribing pilocarpine in the treatment of glaucoma, with particular reference to those patients whose visual field loss approaches the permitted legal minimum for driving. The enhanced effect of pilocarpine in reducing the field of a glaucomatous eye, when compared with a normal eye, may make a significant difference to visual 
function. This may be exacerbated in many elderly patients by the presence of lens opacity. In all patients, the potential visual handicap must be added to the well recognised complications of pilocarpine therapy, and considered in the light of reports in favour of early glaucoma surgery. $22-24$

1 Heiil A. Visual field changes in early glaucoma and how to recognise them. Surv Ophthalmol 1989; 33 (suppl): 403-4.

2 Dunbar Hoskins $H$. Does computerised perimetry offer practical advances in choice of therapy in the glaucoma patient? Eye 1992; 6: 43-6.

3 Mikelberg FS. Do computerised visual fields and automated optic disc analysis assist in the choice of therapy in optic disc analysis assist

4 Lindenmuth KA, Skuta GL, Rabbani R, Musch DC. Effects of pupillary constriction on automated perimetry in norma patients. Ophthalmology 1989; 96: 1298-301.

5 McCluskey DJ, Douglas JP, O'Connor PS, Story K, Ivy LM Harvey JS. The effect of pilocarpine on the visual field in normals Ophthalmology 1986; 93: 843-6.

6 Sanabria O, Feuer WJ, Anderson DR. Pseudo-loss of fixation in automated perimetry. Ophthalmology 1991; 98: 76-8.

7 Engel S. Influence of a constricted pupil on the field in glaucoma. Arch Ophthalmol 1942; 27 : 1184-7.

8 Forbes $M$. Influence of miotics on visual fields in glaucoma. Invest Ophthalmol 1966; 5: 139-45.

9 Rebolleda G, Munoz FJ, Fernandez VJM, Pellicer T, de Castilla JM. Effects of pupillary dilation on automated perimetry in glaucoma patients rceiving pilocarpine. Ophthalmology 1992; 99: 418-23.

10 Lindenmuth KA, Skuta GL, Rabbani R, Musch DC, Bergstrom TJ. Effects of pupillary dilation on automated perimetry in normal patients. Ophthalmology 1990; 97: 367perime.
11 Tate GW Jr, Lynn JR. Principles of quantitative perimetry: testing and interpreting the visual field. New York: Grune and Stratton, 1977: 69.

12 Jay BS. The effective pupillary area at varying perimetric angles. Vision Res 1961; 1: 418-24.

13 Sping KH, Stiles WS. Apparent shape and size of the pupil viewed obliquely. $\mathrm{Br}$ f Ophthalmol 1948; 32: 347-54.

14 Klewin KM, Dadius RL. Background illumination and automated perimetry. Arch Ophthalmol 1986; 104: 395-7.

15 Quigley HA, Dunkelberger GR, Green WR. Retinal ganglion cell atrophy correlated with automated perimetry in huma eyes with glaucoma. Am f Ophthalmol 1989; 107: 453-64.

16 Lam BL, Alward WLM, Kolder HE. Effect of cataract on automated perimetry. Ophthalmology 1991; 98: 1066-70.

17 Katz J, Sommer A. A longitudinal study of the age-adjusted variability of automated visual fields. Arch Ophthalmol 1987; 105: $1083-6$.

18 Heijl A, Lindgren A, Lindgren G. Test-retest variability in glaucomatous visual fields. Am 7 Ophthalmol 1989; 108 . 130-5.

19 Flammer J, Drance SM, Zulauf $M$. Short- and long-term fluctuations in patients with glaucoma, normal controls, and patients with suspected glaucoma. Arch Ophthalmol 1984

20 Bland JM, Altman DG. Statistical methods for assessing agreement between two methods of clinical measurement. Lancet 1986; i: 307-10.

21 Gill JS, Zezulka AV, Beevers DG, Davies P. Relation between initial blood pressure and its fall with treatment. Lancet 1985; i: 567-9.

22 Jay JL, Murray SB. Early trabeculectomy vs conventiona management in primary open angle glaucoma. $\mathrm{Br} F$ Ophthalmol 1988; 72: 881-9.

23 Jay JL, Allan D. The benefit of early trabeculectomy versus conventional management in primary open angle glaucoma relative to severity of disease. Eye 1989; 3: 528-35.

24 Lavin MJ, Wormald RFL, Migdal CS, Hitchings RA. The influence of prior therapy on the success of trabeculectomy. Arch Ophthalmol 1990; 108: 1543-8. 